Geometry $\mathcal{E}$ Topology

Volume 5 (2001) 267-285

Published: 24 March 2001

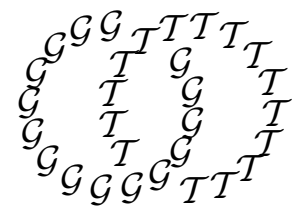

\title{
Homotopy K3's with several symplectic structures
}

\author{
Stefano Vidussi \\ Department of Mathematics, University of California \\ Irvine, California 92697,USA \\ Email: svidussi@math.uci.edu
}

\begin{abstract}
In this note we prove that, for any $n \in \mathbb{N}$, there exist a smooth 4-manifold, homotopic to a $K 3$ surface, defined by applying the link surgery method of Fintushel-Stern to a certain 2-component graph link, which admits $n$ inequivalent symplectic structures.
\end{abstract}

\section{AMS Classification numbers Primary: 57R57}

Secondary: $57 \mathrm{R} 15,57 \mathrm{R} 17$

Keywords: Symplectic topology, 4-manifolds, Seiberg-Witten theory

Proposed: Ronald Fintushel

Seconded: Robion Kirby, Ronald Stern

(C) Geometry ${ }^{\mathcal{G}}$ Topology Publications
Received: 12 December 2000

Revised: 19 February 2001 


\section{Introduction}

Let $M$ be a smooth, closed, oriented 4-manifold. A symplectic form $\omega$ on $M$ is a closed 2-form such that $\omega \wedge \omega>0$. To each symplectic form $\omega$ we can associate a homotopy class of almost complex structures on $M$, with canonical class $K_{\omega} \in H^{2}(M, \mathbb{Z})$.

There exist natural equivalence relations among symplectic forms: Two symplectic forms $\omega_{0}, \omega_{1}$ on $M$ are said to be homotopy equivalent if there exist a path of symplectic forms $\omega_{t}$ connecting them. They are said to be equivalent up to diffeomorphism if there exist a diffeomorphism $f: M \rightarrow M$ such that $f^{*} \omega_{0}=\omega_{1}$. We will say that $\omega_{0}, \omega_{1}$ are equivalent if they are connected by a combination of homotopies and diffeomorphisms. This amounts to saying that, if we denote by $\operatorname{Sympl}(M)$ the space of symplectic forms on the smooth manifold $M$, two symplectic forms are equivalent if and only if they represent elements lying in the same connected component of $\operatorname{Sympl}(M) / \operatorname{Diff}(M)$.

Homotopies preserve the canonical class, while diffeomorphisms act by pull-back on it; these properties can be used to distinguish symplectic structures.

McMullen and Taubes [6] provided the first example of a smooth 4-manifold (together with some generalizations) admitting two inequivalent symplectic structures. The author has then proved, in [9], the "accidental" existence of a third one. These structures are distinguished from the combinatorially inequivalent position of their canonical class in the convex hull of basic classes. Since the appearance of that paper, other examples have been pointed out: in [4] some non-simply connected complex surfaces (with the reversed orientation) are shown to admit two inequivalent structures.

Two points seem to be worthy of further investigation: the first is to exhibit a manifold with inequivalent structures, having "simple topology" (among the simply connected examples quoted above, the simplest is homeomorphic to the elliptic surface $E(4)$ ); the second is to establish the existence, for any $n$, of a manifold admitting $n$ inequivalent structures, possibly in a fixed homeomorphism class.

The aim of this paper is to combine the two points, proving the following theorem:

Theorem 1.1 For any $n \in \mathbb{N}$ there exist a smooth 4-manifold, homotopic (hence homeomorphic) to a $K 3$ surface, admitting $n$ inequivalent symplectic 
forms. ${ }^{1}$

We outline now the idea of the proof. Following the approach of [6] (and, implicitly, of [8]) we start by constructing a family of 2-component links admitting several fibrations over the circle. Fibered 3-manifolds are closely related to symplectic 4-manifolds. The fibrations partition in equivalence classes (the definition of equivalent fibrations, as we will see, follows closely that of symplectic forms). We will study these equivalence classes by analyzing the Euler classes of the fibrations (which play the same role as the canonical classes for symplectic structures). Euler classes of fibrations are vertices of the polyhedron dual to the unit ball of the Thurston norm for the link exterior (see [8]). Different Euler classes correspond to non-homotopic fibrations, and the invariance of the Thurston (or Alexander) norm allows to control equivalence under diffeomorphism.

Next, we fix a link with (at least) $n$ inequivalent fibrations. Using a suitable generalization of the link surgery construction of Fintushel-Stern, we will exhibit, in correspondence to each fibration, a homotopy $K 3$ naturally admitting a symplectic structure, whose canonical class is related to the Euler class of that fibration. The smooth structure of these symplectic manifolds, instead, will not depend on the fibration but only on the link. The underlying smooth manifold can be therefore endowed of symplectic structures which have different canonical classes, hence non-homotopic. There is not a natural analogue of the Thurston norm in dimension 4 , but there is a good analogue of the Alexander norm, related to Seiberg-Witten invariants. We will use the invariance of this suitably defined Seiberg-Witten norm, in order to discuss the obstructions to the equivalence under diffeomorphism.

The paper is divided as follows: Section 2 contains the construction of the family of links we will use, and the study of their fibrations. The first part of this Section is a specialization or a consequence of the general discussion of graph links contained in [1]. In Section 3 we will define, for each link, the (generalized) link surgery 4-manifold, while in Section 4 we will define, by symplectic fiber sum, the symplectic structures on the link surgery manifolds, and discuss their inequivalence. Section 5 contains two examples, and some remarks on the divisibility of the canonical classes.

\footnotetext{
${ }^{1}$ Ivan Smith kindly informed us of his recent and independent construction of a family $X_{n}, n \geq 2$, of pairwise non-homeomorphic simply connected 4-manifolds whose $n$-th member admits $n$ inequivalent structures distinguished by the divisibility of their canonical class (I Smith, On Moduli Space of Symplectic Forms, Math. Res. Lett., to appear).
} 
On the manifolds considered in this paper (as on the previously known examples) symplectic structures are distinguished by comparing their canonical class. It is a major open question whether there exist inequivalent symplectic structures with the same canonical class. A negative answer to this question would imply, by standard results on the SW basic classes, that the number of inequivalent symplectic structures on a fixed smooth manifold is finite.

\section{A class of graph links admitting inequivalent fi- brations}

The aim of this Section is to exhibit a family of 2-component links which admit several fibrations over the circle, and whose Alexander and Thurston norms can be easily computed. Thanks to the thorough analysis of graph links, contained in [1], we can choose among them a family of 2-component graph links which will give us the suitable examples. For reasons that will appear clear later on, we look for links with odd linking number.

Let's introduce the family we will work with. Consider the Seifert fibration of $S^{3}$ with Seifert invariants $(3,1)$ and take the oriented 2-components link $K^{(1)}$ given by two regular fibers with their natural orientation. This is given by the unknot $K_{0}^{(1)}$ and its $(1,3)$-cable $K_{1}^{(1)}$ (order is clearly irrelevant) and is represented, in the notation of [1], by the diagram on the left in Figure 1. This diagram requires some explanation. The node $H$ (a white disk) repre-
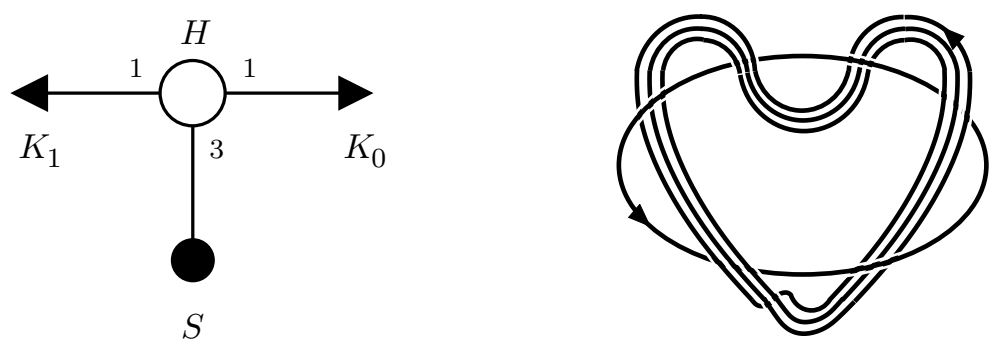

Figure 1: Diagram representation for $K^{(1)}$ (left) and the link $K^{(2)}$ (right)

sents the Seifert fibration of the link exterior; the boundary vertex $S$ (the black disk) represents the tubular neighborhood of the singular fiber of that fibration; the arrowhead vertices denote instead the link components $K_{0}^{(1)}, K_{1}^{(1)}$. Edge weights represent the order of the corresponding fiber: for example, for $K^{(1)}$, the arrowheads are regular fibers (order 1 ), and the boundary vertex is a 
multiple fiber of order 3. The node (interpreted as regular fiber of the Seifert fibration) and the boundary vertex represent, in some sense, virtual components of the link (ie, knots in $S^{3}$ which are not actual components of the link, but are naturally associated to its description in terms of the canonical decomposition of its exterior).

Next, take another copy of the same link and splice the two links along the $K_{0}$ 's (ie, remove a neighborhood of the $K_{0}$ 's and glue the link exteriors identifying meridians with longitudes), obtaining this way a 2 -component link (of linking number 9) in $S^{3}$. Such an operation correspond to the substitution of the component we are splicing with its $(3,1)$-cable ([1], Proposition 9.1). The image of the link is given on the right of Figure 1. Splicing is represented, in diagrams, by joining the edges corresponding to the link components we are splicing.

Iterate $2 n$ times this construction, so to obtain the 2-component graph link $K^{(2 n)} \subset S^{3}$. This is a solvable link (link obtained from unknots by cabling and summing), as the two components are unknots related by the iterated cabling operations; by construction, it has the minimal splice diagram appearing in Figure 2 (minimality refers to the smallest number of edges among equivalent diagrams, as described in Theorem 8.1 of [1]).

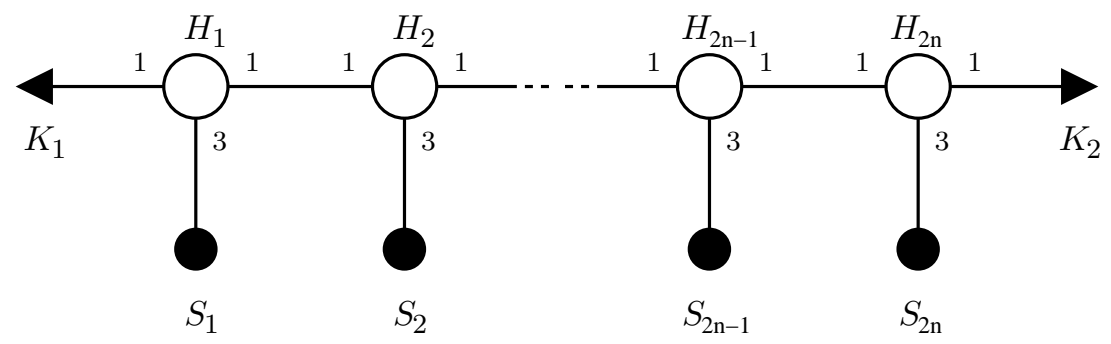

Figure 2: Diagram of the splice decomposition of $K^{(2 n)}$

In this diagram, each node represents a Seifert fibration appearing in the JSJ decomposition of the link exterior, the boundary vertices represent the tubular neighborhood of a singular fiber of that fibration. Together, these represent the virtual components of the link; the arrowhead vertices denote instead the real link components $K_{1}, K_{2}$, in our case regular fibers of the first and last Seifert fibrations. The linking number of this link is $3^{2 n}$. This can be computed from the diagram of Figure 2 by multiplying the edge weights adjacent to the nodes contained in the path joining $K_{1}$ and $K_{2}$ ([1], Theorem 10.1). 
We will denote by $t_{i}$ the homology class of the meridians to the components $K_{i}$. These are the generators of the first homology group of the link exterior $M^{(2 n)}:=S^{3} \backslash \nu K^{(2 n)}$ (with the symbol $\nu(\cdot)$ we will denote the open neighborhood of an embedded submanifold).

As mentioned above, we are interested in the fibrations of the link exterior: we remind the reader that a cohomology class $\mathbf{m}=\left(m_{1}, m_{2}\right) \in H^{1}(M, \mathbb{Z})=$ $\left[M, S^{1}\right]$ is said to be fibered (a fibered multilink, in the notation of [1]) if there exist a fibration of $M$ over $S^{1}$ whose fibers are minimal genus Seifert surfaces representing $\mathbf{m}$, ie, minimal genus representatives of $\mathbf{m}$ transverse to the boundary and intersecting each boundary component in a cable of the link component, whose coefficients and multiplicity are determined by $\mathbf{m}$ according to the formula

$$
\sigma_{i}=-\left(\sum_{j \neq i} m_{j} \cdot l k\left(K_{j}, K_{i}\right)\right) \mu\left(K_{i}\right)+m_{i} \lambda\left(K_{i}\right)
$$

In [1] the authors have analyzed in generality the fiberability, the Thurston norm and the Alexander polynomial of graph links: if we specialize their discussion to the case of the link $K^{(2 n)}$ we have the following result:

Proposition 2.1 (a) Let $\mathbf{m}:=\left(m_{1}, m_{2}\right)$ be a class of $H^{1}\left(M^{(2 n)}, \mathbb{Z}\right)=\mathbb{Z}^{2}$; then $\mathbf{m}$ is fibered if and only if its coordinates lie in the complement of the intersection of $\mathbb{Z}^{2}$ with the $2 n$ lines of equation

$$
3^{i} \cdot m_{1}+3^{2 n-i+1} \cdot m_{2}=0, \quad 1 \leq i \leq 2 n ;
$$

as a consequence, the Thurston and the Alexander norms of $M^{(2 n)}$ coincide.

(b) $\forall 1 \leq i \leq n$ the Thurston norm of the primitive element $P_{i}$ of the $i$-th line, of Cartesian coordinates $\left(3^{2 n+1-2 i},-1\right)$, is given by

$$
\left\|P_{i}\right\|_{T}=3^{4 n-2 i+1}+3^{2 n-2 i+1}+3^{2 n}-2 \cdot 3^{2 n-i}-2 \cdot 3^{2 n-i+1}+1 ;
$$

$\forall n+1 \leq i \leq 2 n$ the Thurston norm of the primitive element $Q_{i}$ of the $i$-th line, of Cartesian coordinates $\left(1,-3^{2 i-1-2 n}\right)$, is given by

$$
\left\|Q_{i}\right\|_{T}=3^{2 i-1}+3^{2 i-2 n-1}+3^{2 n}-2 \cdot 3^{i}-2 \cdot 3^{i-1}+1 ;
$$

$\forall 1 \leq i \leq n$ the indexed family $a_{i}=\left\|P_{i}\right\|_{T}$ is strictly decreasing with $i$ and we have the equality

$$
\left\|P_{i}\right\|_{T}=\left\|Q_{2 n+1-i}\right\|_{T}
$$

(c) The (symmetrized) Alexander polynomial of $K^{(2 n)}$ is given by

$$
\Delta_{K^{(2 n)}}\left(t_{1}, t_{2}\right)=\prod_{1}^{2 n}\left(t_{1}^{3^{i-1}} t_{2}^{3^{2 n-i}}+1+t_{1}^{-3^{i-1}} t_{2}^{-3^{2 n-i}}\right)
$$


Proof The proof of this Proposition follows from Theorems 11.1, 11.2 and 12.1 of [1]. The input we need for applying these Theorems is the linking matrix between the link components $K_{j}$ and the virtual link components corresponding to the regular fiber $H_{i}$ and the singular fiber $S_{i}$ of each Seifert fibration appearing in the minimal splice diagram of $K^{(2 n)}$, see Figure 2. As we chose carefully all the Seifert invariants to be equal, the linking matrix with the $i$-th splice component has the relatively simple form

$$
\left(\begin{array}{cc}
l k\left(K_{1}, H_{i}\right) & l k\left(K_{2}, H_{i}\right) \\
l k\left(K_{1}, S_{i}\right) & l k\left(K_{2}, S_{i}\right)
\end{array}\right)=\left(\begin{array}{cc}
3^{i} & 3^{2 n-i+1} \\
3^{i-1} & 3^{2 n-i}
\end{array}\right) ;
$$

the reader familiar with the content of [1] can read this result from the diagram in Figure 2 above, as linking numbers coincide with the product of the edge weights adjacent to the nodes contained in the path from $H_{i}$ (or $S_{i}$ ) to $K_{j}$ but not on the path itself.

To prove part (a), we recall that Theorem 11.2 of [1] claims that $\mathbf{m}$ is fibered if and only if its coefficients satisfy

$$
\sum_{j=1}^{2} m_{j} \cdot l k\left(K_{j}, H_{i}\right) \neq 0 ; \quad \sum_{j=1}^{2} m_{j} \cdot l k\left(K_{j}, S_{i}\right) \neq 0, \quad \forall 1 \leq i \leq 2 n .
$$

Substituting with the result of Equation 7 we obtain the statement in (a) above. All the $4 n$ (top dimensional) faces of the Thurston unit ball are consequently fibered, ie, all the integral point laying in the cone over these faces are represented by fibrations. It is well known (see eg [5]) that in this case the Thurston and Alexander norm coincide.

Concerning (b), in Theorem 11.1 of [1] it is proven the Thurston norm of any element $\mathbf{m}=\left(m_{1}, m_{2}\right)$ of $H^{1}\left(M^{(2 n)}, \mathbb{Z}\right)$ is given by

$$
\begin{aligned}
& \left\|\left(m_{1}, m_{2}\right)\right\|_{T}= \\
& \quad \sum_{v=1}^{2 n}\left(\delta_{v}-2\right)\left|\sum_{j=1}^{2} m_{j} \cdot\right| k\left(K_{j}, H_{v}\right)\left|+\sum_{w=1}^{2 n}\left(\delta_{w}-2\right)\right| \sum_{j=1}^{2} m_{j} \cdot l k\left(K_{j}, S_{w}\right) \mid
\end{aligned}
$$

where $\delta_{(\cdot)}$ is the number of incident edges of the virtual component (in our case, 3 for the nodes, 1 for the boundary vertices). Plugging in this formula the coordinates of $P_{i}, Q_{i}$, and using the values of the linking matrix of Equation 7 we obtain Equations 3 and 4 . The rest of (b) follows from these results. Figure 3 gives the example of the Thurston unit ball for $n=2$.

Finally, Theorem 12.2 of [1] computes the Alexander polynomial of the link as 


$$
\begin{aligned}
& \Delta_{K^{(2 n)}}\left(t_{1}, t_{2}\right)= \\
& \prod_{v=1}^{2 n}\left(t_{1}^{l k\left(K_{1}, H_{v}\right)} t_{2}^{l k\left(K_{2}, H_{v}\right)}-1\right)^{\delta_{v}-2} \cdot \prod_{w=1}^{2 n}\left(t_{1}^{l k\left(K_{1}, S_{w}\right)} t_{2}^{l k\left(K_{2}, S_{w}\right)}-1\right)^{\delta_{w}-2} ;
\end{aligned}
$$

by using Equation 7 again and taking the symmetrized polynomial we obtain (c).

Notice that the Alexander polynomial is invariant with respect to the interchange of the two variables. This fact, together with the Blanchfield duality $\Delta_{K^{(2 n)}}\left(t_{1}^{-1}, t_{2}^{-1}\right)=\Delta_{K^{(2 n)}}\left(t_{1}, t_{2}\right)$ is reflected in the symmetry of the nonfibered lines and of the norm of their primitive elements about the diagonals $m_{1}=m_{2}, m_{1}=-m_{2}$ (see eg Figure 3 ).

The three dimensional scene is almost complete now. We need to remind the reader (see the Introduction of [6] for example) that to any real cohomology class of $M^{(2 n)}$ lying on the cone over each of the $4 n$ fibered faces of the Thurston unit ball we can associate a closed, nowhere vanishing representative. The classes that are image of integral cohomology classes are represented by the fibrations of $M^{(2 n)}$ considered above. A fibered face is identified by an element of $H_{1}\left(M^{(2 n)}, \mathbb{Z}\right)$, which is the Euler class of the (measured) foliation defined by closed, nowhere vanishing forms. This class (up to a factor -1 ) is the vertex dual to the fibered face in the dual Thurston polyhedron.

We can define equivalence classes of closed, nowhere vanishing forms in a fashion similar to the one used for symplectic forms: Two forms are said to be equivalent if they are connected by a combination of homotopies (through non-vanishing closed forms) and diffeomorphisms of $M^{(2 n)}$.

We can use the Euler class to study the equivalence classes of forms on $M^{(2 n)}$. Although we will not use directly this equivalence result, the method used in the proof provides the model for the proof of Theorem 1.1. We have:

Theorem 2.2 The moduli space of closed non-vanishing forms on $M^{(2 n)}$ contains at least $n+1$ components.

Proof We start by observing that homotopic forms must lie in the cone over the same fibered face of the Thurston unit ball. We will prove the Theorem by showing that the action of $\operatorname{Diff}\left(M^{(2 n)}\right)$ on $H_{1}\left(M^{(2 n)}, \mathbb{Z}\right)$ partitions the $4 n$ Euler classes in $(n+1)$ different orbits. 
The diffeomorphisms of $M^{(2 n)}$ act over $H^{1}\left(M^{(2 n)}, \mathbb{R}\right)$ through isometries of the Thurston (Alexander) norm, preserving the unit ball; they preserve moreover the lattice of integral points (and consequently, divisibility).

We will leave aside the case of $n=1$, where the statement of the Theorem holds true by direct check of the divisibility of the vertices dual to the fibered faces $\left(t_{1}^{8} t_{2}^{8}\right.$ and $t_{1}^{4} t_{4}^{-4}$ are two inequivalent vertices, that we can easily determine from the Alexander polynomial).

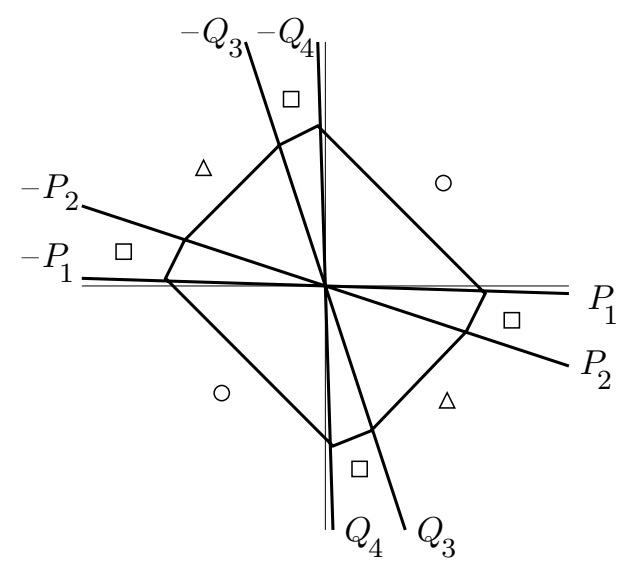

Figure 3: Thurston unit ball for $K^{(4)}$ — non-fibered rays are marked by their primitive element.

Let's assume then that $n \geq 2$; any primitive element $\pm P_{i}, \pm Q_{i}$, lying in one non-fibered ray must be sent to a primitive element lying in a non-fibered ray and having the same norm, ie, $\operatorname{Diff}\left(M^{(2 n)}\right)$ acts on the set of $4 n$ elements $\left\{ \pm P_{j} ; \pm Q_{n+j}, j=1, \ldots, n\right\}$ preserving the norm. Proposition 2.1, (b) implies that there are at least $n$ different orbits for this action: the orbit of $P_{i}$ can contain only $\pm P_{i}, \pm Q_{2 n+1-i}$, as these are the only primitive non-fibered integral homology classes having the same Thurston norm, and the same holds for the other $(4 n-1)$ elements. As $n \geq 2$, the knowledge of the action of $\operatorname{Diff}\left(M^{(2 n)}\right)$ on a single primitive element determines completely the action on the others (eg, if $P_{1}$ goes to $-Q_{2 n}$, then $P_{2}$ must go to $-Q_{2 n-1}$ and so on). This implies a constraint on the possible orbit of fibered faces, and it is easy to check that there must be at least $(n+1)$ orbits of the action of $\operatorname{Diff}\left(M^{(2 n)}\right)$ on the set of fibered faces, the possible identifications corresponding to reflections through the diagonals of $H^{1}\left(M^{(2 n)}, \mathbb{R}\right)$ (the ' +1 ' comes from the fact that the fibered faces hit by the diagonals have orbits containing at most 2 elements, while the orbits of the remaining faces contain at most 4 elements). Figure 3 illustrates 
the case of $n=2$ : the fibered faces marked with a different symbol in Figure are inequivalent. This result implies that the vertices dual to inequivalent fibered faces (and consequently the Euler classes) lie in different orbits of $\operatorname{Diff}\left(M^{(2 n)}\right)$, and completes the proof.

In fact, the existence of inequivalent fibered faces already implies the proof of the Theorem, but as in dimension 4 we will have to work directly with the canonical classes rather than the symplectic forms, we want to emphasize the role of the Euler class.

We want to point out that the idea applied in [6] for proving the inequivalence of the Euler classes, namely the study of the valence of the vertices of the dual Thurston polyhedron, cannot be applied here (nor in the proof of the following Theorem 4.3).

It is conceivable to use direct informations on the Euler classes to deduce their non-equivalence, for example their divisibility. In some specific examples (with small value of $n$ ) it is easy to do so, but for the general case this does not seem to be the simpler approach. Note that to determine explicitly the Euler classes we need only information contained in the Alexander polynomial, to determine the vertices of the convex hull of the Alexander "basic classes" (homology classes

with non-zero coefficient in $\left.\Delta_{K^{(2 n)}}\left(t_{1}, t_{2}\right)\right)$. Or, perhaps more simply, we can observe that the formulae contained in (a) and (b) of Proposition 2.1 allow to determine completely the vertices of the convex hull even without recurring to the Alexander polynomial. We will comment further the question of divisibility in Section 5.

We finish this Section by pointing out that the proof of Theorem 2.2 above holds using both the Thurston norm or the Alexander norm: we will use an analog of the latter in the proof of Theorem 1.1.

\section{Construction of the 4-manifolds}

We recall the definition of a link surgery manifold in this context (for the general case and the details, see [2]): Consider a 2-component oriented link $K \subset S^{3}$ and let $M:=S^{3} \backslash \nu K$. Choose a basis $\left(\alpha_{i}, \beta_{i}\right)$ of simple curves, having intersection 1 , in the homology of the two tori that constitute the boundary of $M$. Next take 2 copies of the rational elliptic surface $E(1)$, containing each one an elliptic fiber $F_{i}$, and define

$$
E(2, K)=\left(\coprod E(1)_{i} \backslash \nu F_{i}\right) \cup_{F_{i} \times S^{1}=S^{1} \times \alpha_{i} \times \beta_{i}} S^{1} \times M
$$


where the gluing is made lifting a diffeomorphism between $S^{1} \times \alpha_{i}$ and $F_{i}$ to an orientation reversing diffeomorphism of the boundary tori in such a way that the homology class of $\beta_{i}$ is sent over the homology class of the normal circle to the $i$-th elliptic fiber. There are usually ambiguities, in the definition of link surgery manifolds, related to the choice of the $\left(\alpha_{i}, \beta_{i}\right)$, the homology basis for the elliptic fiber and the lifting of the diffeomorphism, but in this case, as any orientation preserving diffeomorphism of $\partial(E(1) \backslash \nu F)$ extends to an orientation preserving diffeomorphisms of $(E(1) \backslash \nu F)$ (see [3]), the manifold is well defined and moreover its smooth structure is unaffected by the choice of the homology basis for $\partial M$. The SW polynomial of $E(2, K)$ has been computed in [2] and we have

$$
S W_{E(2, K)}= \pm \Delta_{K}\left(t_{1}^{2}, t_{2}^{2}\right),
$$

where $\Delta_{K}$ is the symmetrized Alexander polynomial of the link, and $t_{i}$ is the image of the class of the meridian $\mu\left(K_{i}\right)$ (that we denoted in the same way) under the injective map

$$
H_{1}(M, \mathbb{Z}) \stackrel{S^{1} \times(\cdot)}{\rightarrow} H_{2}\left(S^{1} \times M, \mathbb{Z}\right) \rightarrow H_{2}(E(2, K), \mathbb{Z}) \stackrel{P D}{\rightarrow} H^{2}(E(2, K), \mathbb{Z}) .
$$

The manifold defined above has the rational homotopy type of $E(2)=K 3$. The Blanchfield duality of the multivariable Alexander polynomial, applied to the two component link, shows that whenever $K$ has odd linking number, then all basic classes are even, so that $E(2, K)$ is a homotopy $K 3$ manifold (in the case at hand, this can also be checked from direct inspection of the $S W$ polynomial).

The family of homotopy $K 3$ 's that will allow us to prove our main result is given, in the smooth category, by $E\left(2, K^{(2 n)}\right)$. We want to point out that these manifolds are not new, in the sense that they are contained in the collection of exotic $K 3$ 's of Fintushel-Stern; their definition in [2] corresponds to the choice of $\left(\alpha_{i}, \beta_{i}\right)=\left(\mu\left(K_{i}\right),-3^{2 n} \mu\left(K_{i}\right)+\lambda\left(K_{i}\right)\right)$, as $l k\left(K_{1}, K_{2}\right)=3^{2 n}$, but any choice gives the same smooth manifold.

\section{Definition of the symplectic structures}

In this Section we will show how we can induce, from inequivalent fibrations on $M^{(2 n)}$, inequivalent symplectic structures to $E\left(2, K^{(2 n)}\right)$. We want to warn the reader that, although inspired by the same bottom line, the idea we will use to construct our examples is slightly different from the one on [6] (in particular, it depends on the use of $E(1))$. We will comment on this at the end of the Section. 
For sake of notation, we will omit the upper index for the link exteriors $M^{(2 n)}$ whenever unnecessary.

Take a fibered cohomology class $\mathbf{m} \in H^{1}(M, \mathbb{Z})$. Recall that such a choice induces a choice (up to isotopy) of a basis element $\beta_{i}$ for the boundary tori of $M$. This is a simple curve determined by the curve $\sigma_{i}$ described in Equation 1 from the relation $\sigma_{i}=d_{i} \beta_{i}$, where $d_{i}>0$ is the divisibility of $\sigma_{i}$. Choose next any simple curve $\alpha_{i}$ in the boundary tori that completes $\beta_{i}$ to a basis, as required in Section 3. The definition of [2], as seen in the previous Section, corresponds to the class $\mathbf{m}=(1,1)$ (so in particular we have necessarily $d_{i}=1$ ) and $\alpha_{i}=\mu\left(K_{i}\right)$.

We can associate to $\mathbf{m}$ a closed three manifold $M(\mathbf{m})$ by Dehn filling the link exterior $M$ with two solid tori $S^{1} \times D^{2}$, using as slope (attaching circle) the two curves $\beta_{i}$. It is a well known fact that this prescription is enough to describe, up to diffeomorphism, the three manifold. This manifold has first Betti number equal to 1 . The fibration $\mathbf{m}$ of $M$ over $S^{1}$ extends to the closed manifold, through the fibration of degree $d_{i}$ of $S^{1} \times D^{2}$ over $S^{1}$ on each solid torus. The cores $C_{i}$ of the solid tori (ie, the so-called dual link in $M(\mathbf{m})$ ) come with a natural framing and clearly satisfy

$$
M=S^{3} \backslash \nu\left(K_{1} \cup K_{2}\right)=M(\mathbf{m}) \backslash \nu\left(C_{1} \cup C_{2}\right) .
$$

Up to isotopy, these cores are transverse to the closed fibers of $M(\mathbf{m})$.

It is a Theorem of Thurston [7] that $S^{1} \times M(\mathbf{m})$ admits a symplectic structure; the embedded, 0 self-intersection tori $S^{1} \times C_{i}$ are symplectic submanifolds of $S^{1} \times M(\mathbf{m})$ (although, if some $d_{i} \neq 1$, they are not sections of the natural fibration over $T^{2}$ ), and come with a trivialization of their normal bundle. We use these submanifolds to present the manifolds $E(2, K)$ as fiber sum: we have the following:

Proposition 4.1 For any choice of fibered $\mathbf{m} \in H^{1}(M, \mathbb{Z})$ we have

$$
E(2, K)=\left(\coprod E(1)_{i}\right) \#_{F_{i}=S^{1} \times C_{i}} S^{1} \times M(\mathbf{m}) .
$$

Proof This is a consequence, as in Section 3, of the property of diffeomorphism extension of $E(1)$; first, the fiber sum in Equation 15 above is well defined, and independent of the choice of the framing for $S^{1} \times C_{i}$; then, because of the diffeomorphism of Equation 14, for all choice of $\mathbf{m}$ the fiber sum manifold is defined as one of those in Equation 11, where we determine the pair of $\beta_{i}$ according to Equation 1 and we complete it to a homology basis of $\partial M$ respecting the framing of $S^{1} \times C_{i}$. Up to diffeomorphism, the smooth manifold defined this way is the same for all $\mathbf{m}$. 
The interest of Proposition 4.1 above stems from the fact that, whenever we perform the fiber sum along symplectic tori, the resulting manifolds admit a symplectic structure.

Our goal is to discuss the equivalence of the symplectic structures arising from different choices of $\mathbf{m}$, as we did for the fibrations in Section 2. In order to do so, we need a norm on homology classes of $E(2, K)$ that plays the same role of the Thurston (Alexander) norm on $M$. We define the Seiberg-Witten norm on $H_{2}(E(2, K), \mathbb{R})=\mathbb{R}^{b}($ with $b=22)$ as

$$
\|\varphi\|_{S W}=\max \left\{k_{i}(\varphi), \quad k_{i} \text { basic class of } E(2, K)\right\} ;
$$

a description of the relation between the SW basic classes and the SW polynomial can be found, eg, in [2]. To study this norm we find convenient to choose a cohomology basis for $H^{2}(E(2, K), \mathbb{Z})$ by taking the first two generators to be $t_{1}, t_{2}$ defined as in Section 3 and completing them to a basis $t_{1}, t_{2}, \ldots, t_{b}$. We take then a dual basis $\Sigma_{j}$ for $H_{2}(E(2, K), \mathbb{Z})$.

For sake of comprehension we can assume that the first two elements of this dual basis are classes whose geometric representatives are defined in the following way (see [2], page 387): consider the two (fibered) classes $H^{1}(M, \mathbb{Z}$ ) of coordinates $(1,0)$ and $(0,1)$. Their Poincaré dual are the classes of the Seifert surfaces of each link component. These have by definition intersection $\delta_{i j}$ with $\mu\left(K_{j}\right)$ and are represented by a genus 0 Riemann surface with $1+3^{2 n}$ discs removed: they span in fact one link component (the first disk) and intersect the tubular neighborhood of the other in $l k\left(K_{1}, K_{2}\right)$ copies of the meridian (the remaining $3^{2 n}$ disks). We can glue these surfaces to the disc sections of $E(1)_{i} \backslash \nu F_{i}$ appearing in the description of $E(2, K)$ (of Equation 15) associated to these two fibered classes. The closed surfaces $\Sigma_{i}$ arising this way satisfy $t_{j}\left(\Sigma_{i}\right)=\delta_{j i}$.

We have now the following simple proposition:

Proposition 4.2 Let $\varphi=m_{1} \Sigma_{1}+m_{2} \Sigma_{2}+\sum_{j=3}^{b} m_{j} \Sigma_{j}=\left(m_{1}, m_{2}, \ldots, m_{b}\right) \in$ $H_{2}(E(2, K), \mathbb{R})$, and denote by $\left(m_{1}, m_{2}\right) \in H^{1}(M, \mathbb{R})$ the element which has the same two first coordinates: then

$$
\|\varphi\|_{S W}=\left\|\left(m_{1}, m_{2}\right)\right\|_{A}=\left\|\left(m_{1}, m_{2}\right)\right\|_{T}
$$

Proof The Seiberg-Witten polynomial of $E(2, K)$ is determined by the Alexander polynomial of $K$ through Equation 12; therefore, the first two coefficients of $\varphi$ determine its SW norm, and that coincides with the Alexander norm of $\left(m_{1}, m_{2}\right)$. 
We note, as a consequence, that the SW unit ball is a cylinder over the SW unit ball on the subspace of $H_{2}(E(2, K), \mathbb{R})$ spanned by $\Sigma_{1}, \Sigma_{2}$, that we will identify now on with $H^{1}(M, \mathbb{R})$. With this identification, the $4 n$ top dimensional (dimension $b-1$ ) faces are a cylinder over the fibered faces of $H^{1}(M, \mathbb{R})$; the cone over each top dimensional face is bounded by the cylinders over two non-fibered rays, cones over lower dimensional (dimension $b-2$ ) faces. We will denote these cylinders using the primitive element of the non-fibered ray, as $C\left( \pm P_{i}\right)$ (or $\left.C\left( \pm Q_{i}\right)\right)$ ); for example, if the coordinates of $P_{i}$ are $\left(m_{1}, m_{2}\right)$, $C\left(P_{i}\right)=\left\{\left(\mu_{1}, \mu_{2}, \ldots, \mu_{b}\right) \in H_{2}\left(E\left(2, K^{(2 n)}\right), \mathbb{R}\right) \mid \mu_{1}=\lambda m_{1}, \quad \mu_{2}=\lambda m_{2}, \quad \lambda>0\right\}$.

Figure 4 illustrates the previous definitions.

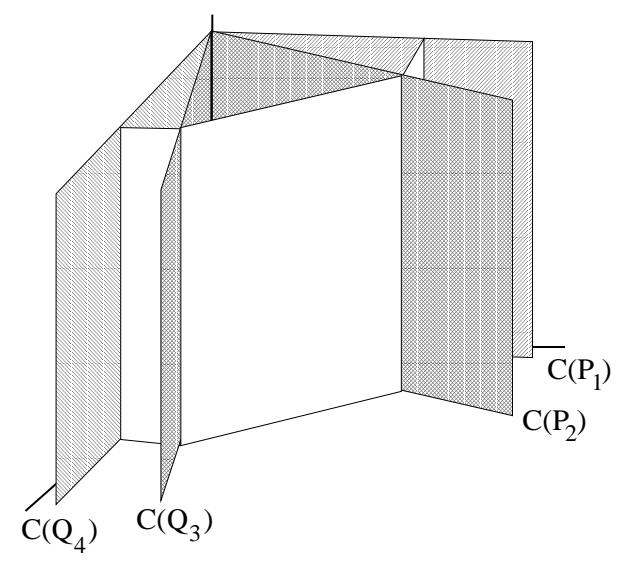

Figure 4: $H_{2}\left(E\left(2, K^{(4)}\right), \mathbb{R}\right)$ - the shaded planes are some of the cylinders over nonfibered rays; the white planes represents some of the top dimensional faces.

With the knowledge of the SW norm of $E(2, K)$ we are ready to state our main result. We have:

Theorem 4.3 (a) For each fibered cohomology classes $\mathbf{m} \in H^{1}(M, \mathbb{Z})$ there exist a symplectic structure on $E(2, K)$ whose canonical bundle is the image (up to a factor -1 ) of the Euler class of the fibration under the injective map $H_{1}(M, \mathbb{Z}) \rightarrow H^{2}(E(2, K), \mathbb{Z})$.

(b) Inequivalent fibrations induce inequivalent symplectic structures on the same smooth manifold $E(2, K)$; in particular, there are $n+1$ inequivalent symplectic structures on $E\left(2, K^{(2 n)}\right)$. 
Proof (a) For all choice of a fibered class $\mathbf{m}$ we have a symplectic manifold $S^{1} \times M(\mathbf{m})$, with a pair of embedded, framed symplectic tori $S^{1} \times C_{i} . E(1)$, with its standard Kähler form, is as well symplectic, with $F$ an embedded, framed symplectic torus. Gompf's theory of symplectic fiber sum (see eg [3]) guarantees that the manifolds defined as in Equation 15 admit a symplectic structure. Proposition 4.1 tells that the smooth manifold carrying the symplectic structure is the same for any $\mathbf{m}$. Once $\mathbf{m}$ is fixed, Theorem 3.4 of [6] allows to identify the canonical class of the symplectic structure on $E(2, K)$ as the image, with reversed sign, of the Euler class of the fibration under the injective map $H_{1}(M, \mathbb{Z}) \rightarrow H^{2}(E(2, K), \mathbb{Z})$ described in Equation 13. In fact, in [6] it is shown that the anticanonical bundle has a section whose zero set is homologous to the image of the Euler class associated to $\mathbf{m}$ cross the class of $S^{1}$. This section is obtained in the following way. The anticanonical bundle restricts on $S^{1} \times M$ to the pull-back of the plane field defined by the fibration $\mathbf{m}$, which has a preferred section which is inward pointing on the boundary and whose zero set is the Euler class of $\mathbf{m}$. This section obviously induces a section of the anticanonical restricted to $S^{1} \times M$. On $E(1) \backslash \nu F$ the anticanonical is a trivial bundle (recall that $K_{E(1)}=-F$ ) and we can construct a nowhere vanishing section by restricting a section of $E(1)$ with a simple zero along $F$ : on the boundary $F \times S^{1}$, the section can be assumed to depend only on the $S^{1}$ coordinate, (as the tangent bundle to the elliptic fiber is canonically trivial), and outward pointing, because of the simple zero at $F$. These two sections glue on the boundary to give a section, on $E(2, K)$, whose zero set is the image of the Euler class under the map of Equation 13.

Note that the canonical classes arising through this construction satisfy (as they should) all Taubes' constraints for $E(2, K)$, as they are image of a vertex of the polyhedron dual to the Alexander norm ball and therefore, by Equation 12, they are the vertices of the convex hull of basic classes.

To prove part (b) we note that, as symplectic forms with different canonical bundles are not homotopic, we can construct at least $4 n$ non-homotopic symplectic forms on $E\left(2, K^{(2 n)}\right)$ by choosing, in the symplectic fiber sum definition of Equation 15, elements $\mathbf{m}$ laying in different fibered cones of $H^{1}\left(M^{(2 n)}, \mathbb{Z}\right)$ (and having thus different Euler classes). We need to prove now that the $4 n$ canonical bundles arising this way partition in at least $(n+1)$ orbits under the action of the diffeomorphism group of $E\left(2, K^{(2 n)}\right)$.

Diffeomorphisms preserve the convex hull of Seiberg-Witten basic classes, as well as the unit ball of the Seiberg-Witten norm, which is its dual polyhedron. We will study the orbits of the vertices of the convex hull (and therefore of the canonical bundles) by studying the orbits of their dual (top dimensional) face. 
Again, we leave aside the case of $n=1$, where we can easily apply divisibility to prove the result.

Let's assume then that $n \geq 2$. We will study the orbits of the classes of $H_{2}\left(E\left(2, K^{(2 n)}\right), \mathbb{R}\right.$ ) laying in the plane spanned by $\Sigma_{1}, \Sigma_{2}$ (which can be identified with $H^{1}\left(M^{(2 n)}, \mathbb{R}\right)$ ) with same coordinates as $\pm P_{i}$ or $\pm Q_{i}$. For sake of notation, we denote these elements with the same symbol. By invariance of the unit ball, diffeomorphisms exchange the cones $\left\{C\left( \pm P_{j}\right), C\left( \pm Q_{n+j}\right), j=\right.$ $1, \ldots, n\}$ : the image of an element $\pm P_{i}, \pm Q_{i}$ must be therefore a primitive element laying in one of the $\left\{C\left( \pm P_{j}\right), C\left( \pm Q_{n+j}\right), j=1, \ldots, n\right\}$ and having the same Seiberg-Witten norm. Note that there is no reason why diffeomorphism should preserve the span of $\Sigma_{1}, \Sigma_{2}$. We need to modify a bit the approach to the proof of Theorem 2.2 to keep track of this.

Consider one of the four primitive classes $\varphi=\left(m_{1}, m_{2}, 0, \ldots, 0\right)$ whose coefficients $m_{1}, m_{2}$ lie on non-fibered rays of $H^{1}\left(M^{(2 n)}, \mathbb{Z}\right)$, and which have the smallest SW norm. According to Prop 2.1 and Proposition 4.2, this must be one of the $\left\{ \pm P_{n}, \pm Q_{n+1}\right\}$. Take $P_{n}$ for example. Under the action of a diffeomorphism $f, \varphi$ is sent to a primitive class $f_{*} \varphi=\left(m_{1}^{\prime}, m_{2}^{\prime}, m_{3}^{\prime}, \ldots, m_{b}^{\prime}\right)$ where, in principle, $\left(m_{1}^{\prime}, m_{2}^{\prime}\right)$ can be divisible, but must be integer multiple of the coordinates of one of the $\left\{ \pm P_{j}, \pm Q_{n+j}, j=1, \ldots, n\right\}$. Moreover we must have $\|\varphi\|_{S W}=\left\|f_{*} \varphi\right\|_{S W}$ : consequently, by Proposition 4.2

$$
\left\|\left(m_{1}, m_{2}\right)\right\|_{T}=\left\|\left(m_{1}^{\prime}, m_{2}^{\prime}\right)\right\|_{T} .
$$

As the norm of $\left(m_{1}, m_{2}\right)$ is minimal, we deduce that in fact $\left(m_{1}^{\prime}, m_{2}^{\prime}\right)$ are the coordinates of one of the $\left\{ \pm P_{n}, \pm Q_{n+1}\right\}$. This condition constrains the orbit of the entire cone $C\left(P_{n}\right) ; f_{*} C\left(P_{n}\right)$ must be one of the four cones $\left\{C\left( \pm P_{n}\right)\right.$, $\left.C\left( \pm Q_{n+1}\right)\right\}$. We can repeat this argument for the other three elements of minimal SW norm, and then pass to the other four of second minimal norm and so on. Or, more simply, we observe that as $n \geq 2$ when we know $f_{*} C\left(P_{n}\right)$ then we can unambiguously determine the image of the other cones (take $Q_{n+1}$ and proceed as above to determine $f_{*} C\left(Q_{n+1}\right)$, and then use the invariance of the unit ball).

It is easy to check that this result implies that there are at least $(n+1)$ orbits of the action of the diffeomorphism group of $E\left(2, K^{(2 n)}\right)$ on the set of $4 n$ top dimensional faces of the unit ball, which play the role of the fibered faces for the link exterior. Again, the possible identifications correspond to reflection of these faces about the diagonals $m_{1}=m_{2}, m_{1}=-m_{2}$.

The dual vertices to these faces, and so the canonical bundles, lie in at least $(n+1)$ distinguished orbits of the group of diffeomorphism. This completes the proof of (b). 
We finish this Section by pointing out the difference between McMullen-Taubes example and ours. In the case of [6], the two inequivalent symplectic structures arise from inequivalent fibrations, on the link complement, obtained as restriction of fibrations over the same closed three manifold. In particular, the link surgery construction of the manifold is the same for all fibrations. In our construction, such approach is not viable, and we are constrained to use different closed three manifold, and different link surgery construction, for each fibration. The extension properties of the diffeomorphisms of $E(1)$ allow to obtain, in spite of this, diffeomorphic manifolds. The nature of our examples, therefore, is in some sense more similar to the situation of the "accidental" existence of a third symplectic structure on the McMullen-Taubes manifold, proven in [9] defining a different link surgery presentation of the same smooth manifold.

\section{Some examples}

In this Section we will work out some details of the calculation for $n=2$ and $n=4$, examples that will allow to make some remarks on the divisibility of the canonical classes. We start with $n=2$; the primitive elements of the 4 nonfibered lines in $H^{1}\left(M^{(4)}, \mathbb{Z}\right)$, together with their Thurston (Alexander) norms, are

$$
\begin{array}{ccc}
P_{1}=\left(3^{3},-1\right), \quad\left\|P_{1}\right\|_{T}=2080 & P_{2}=(3,-1), \quad\left\|P_{2}\right\|_{T}=256 \\
Q_{3}=(1,-3), \quad\left\|Q_{3}\right\|_{T}=256 & Q_{4}=\left(1,-3^{3}\right), \quad\left\|Q_{4}\right\|_{T}=2080 .
\end{array}
$$

From these data we can deduce, as in Section 2, that the three fibered faces contained between $P_{1}$ and $P_{2}, P_{2}$ and $Q_{3},-Q_{4}$ and $P_{1}$ are not equivalent under diffeomorphism (see Figure 3 ). When we extend this argument to the Seiberg-Witten norm on $H_{2}\left(E\left(2, K^{(4)}\right), \mathbb{R}\right)$, as in Section 4 , we obtain the proof of the non-equivalence of the top dimensional faces bounded by $C\left(P_{1}\right)$ and $C\left(P_{2}\right), C\left(P_{2}\right)$ and $C\left(Q_{3}\right), C\left(-Q_{4}\right)$ and $C\left(P_{1}\right)$. The canonical classes dual to these faces determine three inequivalent symplectic structures, as stated in Theorem 4.3.

Let's see how we can obtain the same result with an independent argument: the knowledge of the non-fibered lines and the Thurston norm allows to compute the dual vertex of the fibered face bounded by two non-fibered rays. We will determine, more precisely, the vertices of the convex hull of the Alexander basic classes. If $\left(a_{1}, a_{2}\right)$ and $\left(b_{1}, b_{2}\right)$ are the coordinates of two non-fibered primitive elements bounding a fibered cone (eg, $P_{i}$ and $P_{i+1}, P_{n}$ and $Q_{n+1}$ and so on) 
their Thurston norm is determined by pairing with the same dual vertex of coordinates $(x, y)$, according to the formula

$$
\left(\begin{array}{cc}
a_{1} & a_{2} \\
b_{1} & b_{2}
\end{array}\right)\left(\begin{array}{l}
x \\
y
\end{array}\right)=\frac{1}{2}\left(\begin{array}{c}
\left\|\left(a_{1}, a_{2}\right)\right\|_{T} \\
\left\|\left(b_{1}, b_{2}\right)\right\|_{T}
\end{array}\right) .
$$

From this we can compute the vertices of the convex hull of Alexander basic classes: they are given by the terms

$$
t_{1}^{40} t_{2}^{40}, \quad t_{1}^{38} t_{2}^{-14}, \quad t_{1}^{32} t_{2}^{-32}, \quad t_{1}^{14} t_{2}^{-38}
$$

and the other 4 given by Blanchfield duality.

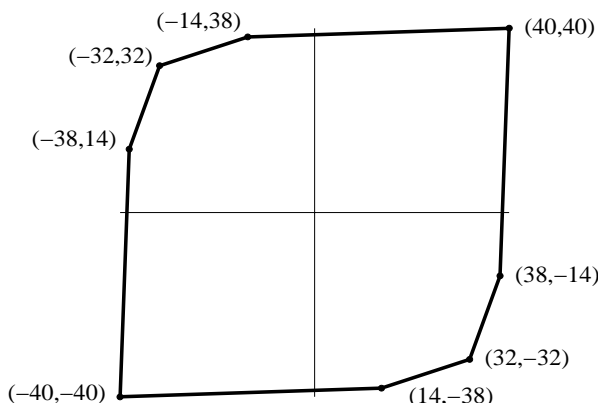

Figure 5: Convex hull of basic classes for $K^{(4)}$

A check of the Alexander polynomial (with the help of a computer algebra system) shows that these are in fact the vertices of the convex hull of the Alexander basic classes, represented in Figure 5. Note that these vertices are orthogonal to the faces of the Thurston unit ball, represented in Figure 3 .

The first three classes of Equation 22 are dual (up to a factor 2) to the three inequivalent faces. But we can observe, in any case, that they have different divisibility, and necessarily cannot be joined by a diffeomorphism. The Euler classes of the fibrations are given by the square (in multiplicative notation) of the classes in Equation 22, and the same is true (with the aforementioned identification of the variables $t_{i}$ as elements of $H^{2}\left(E\left(2, K^{(4)}\right), \mathbb{Z}\right)$, see Equation 12) for the canonical classes corresponding to the inequivalent symplectic structures. In this case, therefore, the inequivalence of the symplectic structures can be proven by the sole computation of the Euler classes of the three dimensional fibrations, and in particular without the use of gauge theory.

The case with $n=4$ appears somewhat different. In that case, we have 8 non-fibered lines, and 16 fibered faces. The discussion of Section 2 leads to 
the identification of 5 inequivalent classes of fibrations. These classes are represented by the faces $\left(P_{1}, P_{2}\right),\left(P_{2}, P_{3}\right),\left(P_{3}, P_{4}\right),\left(P_{4}, Q_{5}\right),\left(-Q_{1}, P_{1}\right)$. The vertices of the convex hull of the Alexander basic classes dual to these faces can be computed, as before, using Equation 21, and turn out to be

$$
t_{1}^{3280} t_{2}^{3280}, t_{1}^{3278} t_{2}^{-1094}, t_{1}^{3272} t_{2}^{-2552}, t_{1}^{3254} t_{2}^{-3038}, t_{1}^{3200} t_{2}^{-3200} .
$$

Among these classes, the second and the fourth have both divisibility 2; a check of the other divisibilities leads to distinguish at most 4 classes, and consequently at most 4 symplectic structures on $E\left(2, K^{(8)}\right)$. The divisibility criterion for canonical bundles appears therefore weaker than the study of the Seiberg-Witten norm, for our family of examples. This result should not appear too surprising: we remind the reader that all the symplectic structures on the McMullen-Taubes manifold defined in [6] and [9] have the same divisibility.

It would be interesting to prove that the number of canonical classes of different divisibility on $E\left(2, K^{(2 n)}\right)$ grows with no bound with $n$. This would give an alternative, non-gauge-theoretic proof of Theorem 1.1.

\section{References}

[1] D Eisenbud, W Neumann, Three Dimensional Link Theory and Invariants of Plane Curve Singularities, Annals of Mathematics Studies 110 (1985)

[2] R Fintushel, R Stern, Knots, Links, and 4-manifolds, Invent. Math. 134 (1998) 363-400

[3] R Gompf, A Stipsicz, 4-Manifolds and Kirby Calculus, Graduate Studies in Mathematics (vol. 20) AMS (1999)

[4] C LeBrun, Diffeomorphisms, Symplectic Forms and Kodaira Fibrations, preprint (2000)

[5] C McMullen, The Alexander Polynomial of a Three Manifold and the Thurston Norm on Cohomology, preprint (1998)

[6] C McMullen, C Taubes, 4-Manifolds with Inequivalent Symplectic Forms and 3-Manifolds with Inequivalent Fibrations, Math. Res. Lett. 6 (1999) 681-696

[7] W Thurston, Some Simple Examples of Symplectic Manifolds, Proc. Amer. Math. Soc. 55 (1976) 467-468

[8] W Thurston, A Norm on the Homology of 3-manifolds, Memoirs of the AMS, 59 (1986) 99-130

[9] S Vidussi, Smooth Structure of Some Symplectic Surfaces, (2000) to appear in Michigan Math. J. 